\title{
General geometry of belief function combination
}

\author{
Fabio Cuzzolin ${ }^{1}$ \\ Oxford Brookes University, UK \\ fabio.cuzzolin@brookes.ac.uk
}

\begin{abstract}
In this paper we build on previous work on the geometry of Dempster's rule to investigate the geometric behaviour of various other combination rules, including Yager's, Dubois', and disjunctive combination, starting from the case of binary frames of discernment. Believability measures for unnormalised belief functions are also considered. A research programme to complete this analysis is outlined.
\end{abstract}

Keywords: Geometry · Yager's and Dubois' combination · conjunctive and disjunctive combination · unnormalised belief functions.

\section{Introduction}

In the geometric approach to uncertainty and belief function theory [3], belief measures are represented as points of a convex space, termed belief space $\mathcal{B}$ [2]. In a series of papers, in particular, this author studied the behaviour of Dempster's rule of combination in this geometric setting [1]. An earlier analysis of Dempster's rule on binary domains can be found in [6].

In this work, we start to extend this geometric analysis to several other major combination operators, including Yager's [10] and Dubois' rules, but also the disjunctive operator [8]. The final objective of the research programme is a comparative geometric analysis of combination rules, which would eventually allow us to describe the 'cone' of possible future belief states under stronger or weaker assumptions on reliability and independence of sources, associated with conjunctive and disjunctive combination. The bulk of the analysis focusses on standard, normalised belief functions - towards the end, however, we also consider unnormalised belief functions [9] and provide some preliminary results.

We start by giving a general definition of conditional subspace (cfr. [3], Chapter 8 ), as the set of possible future states under a given combination rule.

Definition 1. Given a belief function $(B F)$ Bel $\in \mathcal{B}$ we call conditional subspace $\langle B e l\rangle_{\odot}$ the set of all $\odot$ combinations of Bel with any other BF Bel' defined on the same frame, where $\odot$ is an arbitrary combination rule, assuming their combination exists: $\langle\text { Bel }\rangle_{\odot} \doteq\left\{B e l \odot B e l^{\prime}, B e l^{\prime} \in \mathcal{B}\right.$ s.t. $\left.\exists\left(B e l \odot B e l^{\prime}\right)\right\}$.

Our analysis will be conducted on binary spaces, and used to formulate conjectures on the case of general frames of discernment. We will first recall the necessary notions of the geometric approach to belief theory in Section 2. We 
will consider Yager's and Dubois' rules in Section 3, disjunctive combination in Section 4, to cover the behaviour of unnormalised BFs in Section 5. We will draw some verdicts and outline future work in our Conclusions.

\section{Belief functions and their geometry}

Belief functions. A basic probability assignment (BPA) [7] over a discrete set (frame) $\Theta$ is a function $m: 2^{\Theta} \rightarrow[0,1]$ defined on $2^{\Theta}=\{A \subseteq \Theta\}$ such that: $m(\emptyset)=0, \sum_{A \subset \Theta} m(A)=1$. The belief function (BF) associated with a BPA $m$ : $2^{\Theta} \rightarrow[0,1]$ is the function $\operatorname{Bel}: 2^{\Theta} \rightarrow[0,1]$ defined as: $\operatorname{Bel}(A)=\sum_{B \subseteq A} m(B)$. The elements of the power set $2^{\Theta}$ associated with non-zero values of $m$ are called the focal elements of $m$. For each subset ('event') $A \subset \Theta$ the quantity $\operatorname{Bel}(A)$ is called the degree of belief that the outcome lies in A. Dempster's combination $\mathrm{Bel}_{1} \oplus \mathrm{Bel}_{2}$ of two belief functions on $\Theta$ is the unique BF there with as focal elements all the non-empty intersections of focal elements of $B e l_{1}$ and $B e l_{2}$, and basic probability assignment: $m_{\oplus}(A)=\frac{m_{\cap}(A)}{1-m_{\cap}(\emptyset)}$, where $m_{\cap}(A)=$ $\sum_{B \cap C=A} m_{1}(B) m_{2}(C)$ and $m_{i}$ is the BPA of the input BF $B e l_{i}$.

Belief space. Given a frame of discernment $\Theta$, a BF Bel is specified by its $N-2$ belief values $\{\operatorname{Bel}(A), \emptyset \subsetneq A \subsetneq \Theta\}, N \doteq 2^{|\Theta|}$, and can then be represented as a point of $\mathbb{R}^{N-2}$. The belief space $[1,2]$ associated with $\Theta$ is the set of points $\mathcal{B}$ of $\mathbb{R}^{N-2}$ which correspond to proper belief functions. It can be proven that the belief space $\mathcal{B}$ is the convex closure $C l$ of all the vectors associated with categorical $\mathrm{BFs} \mathrm{Bel}_{A}$ (such that $\left.m(A)=1\right): \mathcal{B}=C l\left(B_{e l}, \emptyset \subsetneq A \subseteq \Theta\right)=$ $\left\{\sum_{\emptyset \subseteq A \subseteq \Theta} \alpha_{A} B e l_{A}, \alpha_{A} \geq 0 \forall A, \sum_{A} \alpha_{A}=1\right\}$, an $(N-2)$-dimensional simplex.

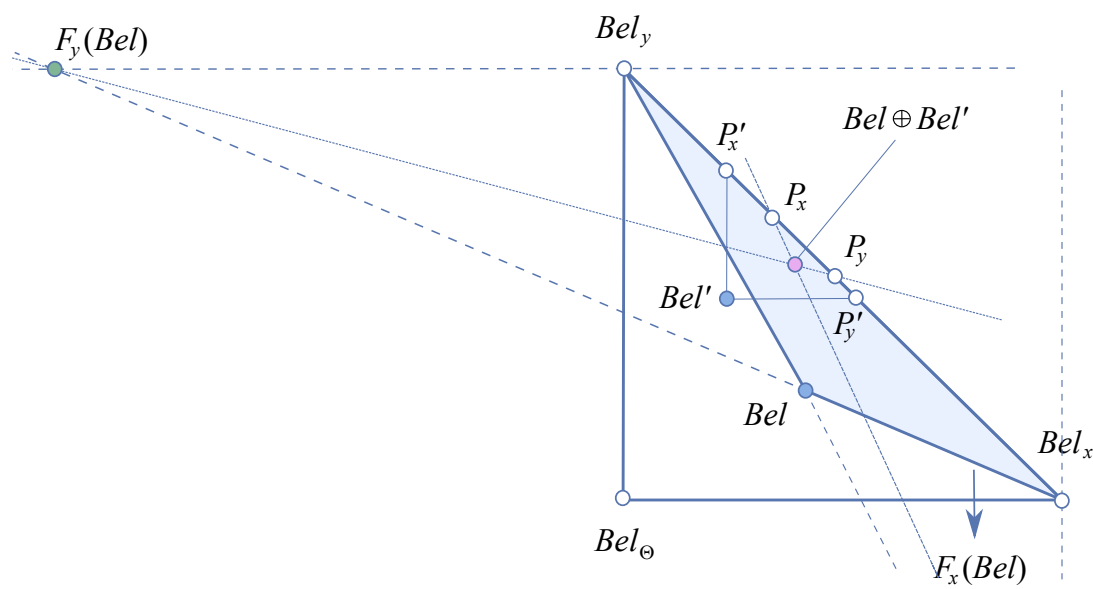

Fig. 1. Graphical construction of Dempster's combination in the binary belief space.

Geometry of Dempster's rule. In [3] we proved that the conditional subspace $\langle B e l\rangle$ under Dempster's combination is $\langle B e l\rangle=C l\left\{B e l \oplus \mathrm{Bel}_{A}, A \subseteq \mathcal{C}_{B e l}\right\}$, where $\mathcal{C}_{B e l}$ is the union of the focal elements of $\mathrm{Bel}$ (see Figure 1, in light blue, for the binary case $\left.\Theta_{2}=\{x, y\}\right)$. Dempster's combination of a BF Bel with 
another BF Bel $^{\prime}$ with mass $m^{\prime}$ describes, for $m^{\prime}(y) \in \mathbb{R}^{1}$, a straight line in the belief space, except the point with coordinates: $F_{x}(B e l)=\left[1,-\frac{m\left(\Theta_{2}\right)}{m(x)}\right]^{\prime},{ }^{2}$ which coincides with the limit of $\mathrm{Bel} \oplus \mathrm{Bel}^{\prime}$ for $m^{\prime}(y) \rightarrow \pm \infty$. This is true for every value of $m^{\prime}(x) \in[0,1]$. Indeed, all the collections of Dempster's sums $\mathrm{Bel} \oplus \mathrm{Bel}^{\prime}$ with $m^{\prime}(x)=k=$ const have a common intersection at the point $F_{x}(B e l)$, which is located outside the belief space. In the same way, this holds for the sets $\left\{\mathrm{Bel} \oplus \mathrm{Bel}^{\prime}: \mathrm{m}^{\prime}(y)=l=\right.$ const $\}$, which each form a distinct line passing through a twin point: $F_{y}(\mathrm{Bel})=\left[-\frac{m(\Theta)}{m(y)}, 1\right]^{\prime}$.

We call $F_{x}(\mathrm{Bel}), F_{y}(\mathrm{Bel})$ the foci of the conditional subspace $\langle\mathrm{Bel}\rangle$.

Dempster's rule thus admits an elegant geometric construction in the belief space, illustrated, for the binary case, in Figure 1.

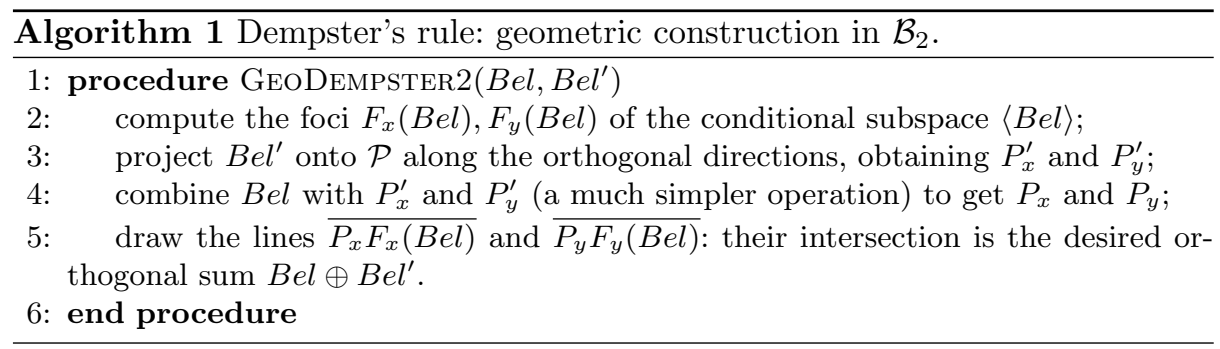

These notions can be naturally extended to finite frames with an arbitrary number $|\Theta|$ of elements ([3], Chapter 8).

\section{Geometry of Yager's and Dubois' rules}

Yager's and Dubois' rules. Yager's rule [10] is based on the view that conflict is generated by non-reliable information sources. In response, the conflicting mass (here denoted by $\left.m_{\cap}(\emptyset)\right)$ is re-assigned to the whole frame of discernment $\Theta$ :

$$
m_{\odot}(A)= \begin{cases}m_{\cap}(A) & \emptyset \neq A \subsetneq \Theta \\ m_{\cap}(\Theta)+m_{\cap}(\emptyset) A=\Theta .\end{cases}
$$

The combination operator proposed by Dubois and Prade [5] comes from applying the minimum specificity principle to the cases in which the focal elements $B, C$ of two input BFs do not intersect, and assigns their product mass to $B \cup C$ :

$$
m_{D}(A)=m_{\cap}(A)+\sum_{B \cup C=A, B \cap C=\emptyset} m_{1}(B) m_{2}(C) .
$$

Analysis on binary frames. On binary frames, $\Theta=\{x, y\}$ Yager's rule (1) and Dubois' rule (2) coincide, as the only conflicting focal elements are $\{x\}$ and

\footnotetext{
${ }^{1}$ For Dempster's rule can be extended to pseudo belief functions.

${ }^{2}$ We write $m(x)$ instead of $m(\{x\}), B e l_{x}$ rather than $B e l_{\{x\}}$ to simplify the notation.
} 
$\{y\}$, whose union is $\Theta$ itself:

$$
\begin{aligned}
& m_{\Theta}(x)=m_{1}(x)\left(1-m_{2}(y)\right)+m_{1}(\Theta) m_{2}(x), \\
& m_{\Theta}(y)=m_{1}(y)\left(1-m_{2}(x)\right)+m_{1}(\Theta) m_{2}(y), \\
& m_{\Theta}(\Theta)=m_{1}(x) m_{2}(y)+m_{1}(y) m_{2}(x)+m_{1}(\Theta) m_{2}(\Theta) .
\end{aligned}
$$

Using (3) we can easily show that:

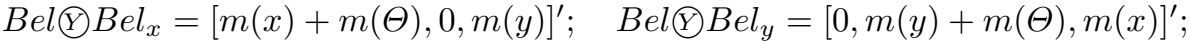

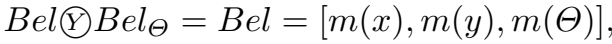

once adopting the vector notation $\operatorname{Bel}=[\operatorname{Bel}(x), \operatorname{Bel}(y), \operatorname{Bel}(\Theta)]^{\prime}$.

The conditional subspace $\langle\mathrm{Bel}\rangle_{\otimes}$ (Figure 2 (left)) is thus the convex closure of the points (4): $\langle\mathrm{Bel}\rangle_{\odot}=\mathrm{Cl}\left(\mathrm{Bel}, \mathrm{Bel}\left(\mathrm{Bel}_{x}, \mathrm{Bel}_{(}\left(\mathrm{Bel}_{y}\right)\right.\right.$.
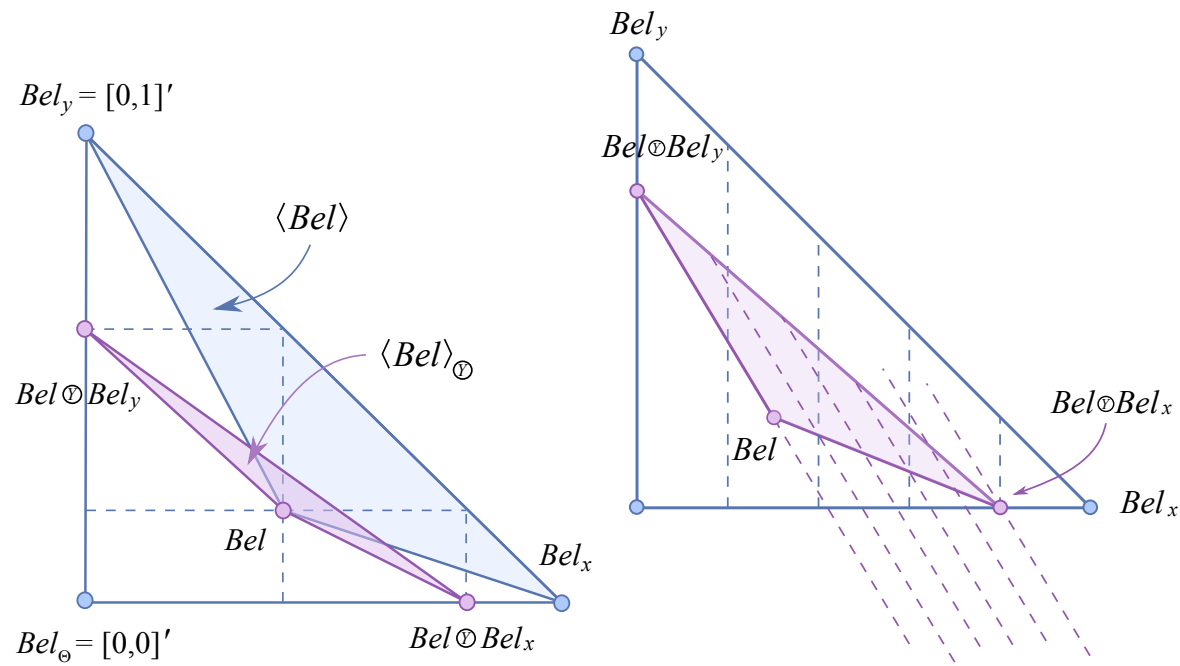

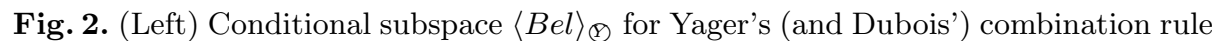
on a binary frame $\Theta=\{x, y\}$. Dempster's $\langle B e l\rangle$ is also shown for comparison. (Right) In Yager's combination, the images of constant mass loci (dashed blue segments) do not converge to a focus, but form parallel lines (dashed purple, cfr. Figure 1).

Comparing (3) with (4), it is easy to see that

$$
\mathrm{Bel}_{1}(\mathrm{P}) \mathrm{Bel}_{2}=m_{2}(x)\left(\mathrm{Bel}_{1}\left(\mathrm{P} B \mathrm{Be}_{x}\right)+m_{2}(y)\left(\mathrm{Bel}_{1}(\mathrm{Y}) \mathrm{Bel}_{y}\right)+m_{2}(\Theta)\left(\mathrm{Bel}_{1}\left(\mathrm{Bel}_{\Theta}\right),\right.\right.
$$

i.e., the simplicial coordinates of $B \mathrm{Bl}_{2}$ in the binary belief space $\mathcal{B}_{2}$ and of the Yager combination $B e l_{1}\left(\mathrm{~B} B e l_{2}\right.$ in the conditional subspace $\left\langle B e l_{1}\right\rangle_{(}$coincide. We can then conjecture the following.

Conjecture 1. Yager combination and affine combination commute. Namely:

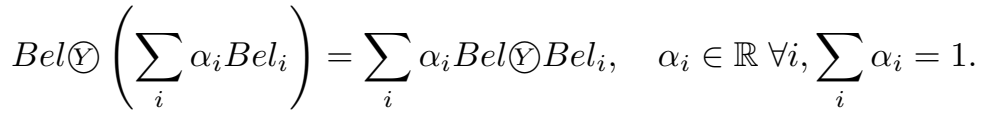


As commutativity is the basis for the geometric analysis of Dempster's rule [1], this opens the way for a similar geometric construction for Yager's rule. However, as shown in Figure 2 (right), images of constant mass loci under Yager's rule are parallel, and there are no foci. From (3) it follows that:

$$
\lim _{m_{2}(y) \rightarrow-\infty} \frac{m_{\Theta}(y)}{m_{\Theta}(x)}=\frac{m_{1}(y)\left(1-m_{2}(x)\right)+m_{1}(\Theta) m_{2}(y)}{m_{1}(x)\left(1-m_{2}(y)\right)+m_{1}(\Theta) m_{2}(x)}=-\frac{m_{1}(\Theta)}{m_{1}(x)},
$$

and similarly for the loci with $m_{2}(y)=$ const.

Nevertheless, as we will rigorously prove in upcoming work, Yager's combination also admits a geometric construction based on intersecting linear spaces which are images of constant mass loci.

\section{Geometry of disjunctive combination}

Disjunctive combination [8] is the natural, cautious dual of Dempster's combination. The operator follows from the assumption that the consensus between two sources of evidence is best represented by the union of the supported hypotheses, rather than by their intersection. An algebraic analysis of disjunctive combination on binary frames, in the form of 'Dempster semigroups', is due to Daniel [4]. Combination results are there visualised in a way similar to that presented here, although the focus is not on the geometry.
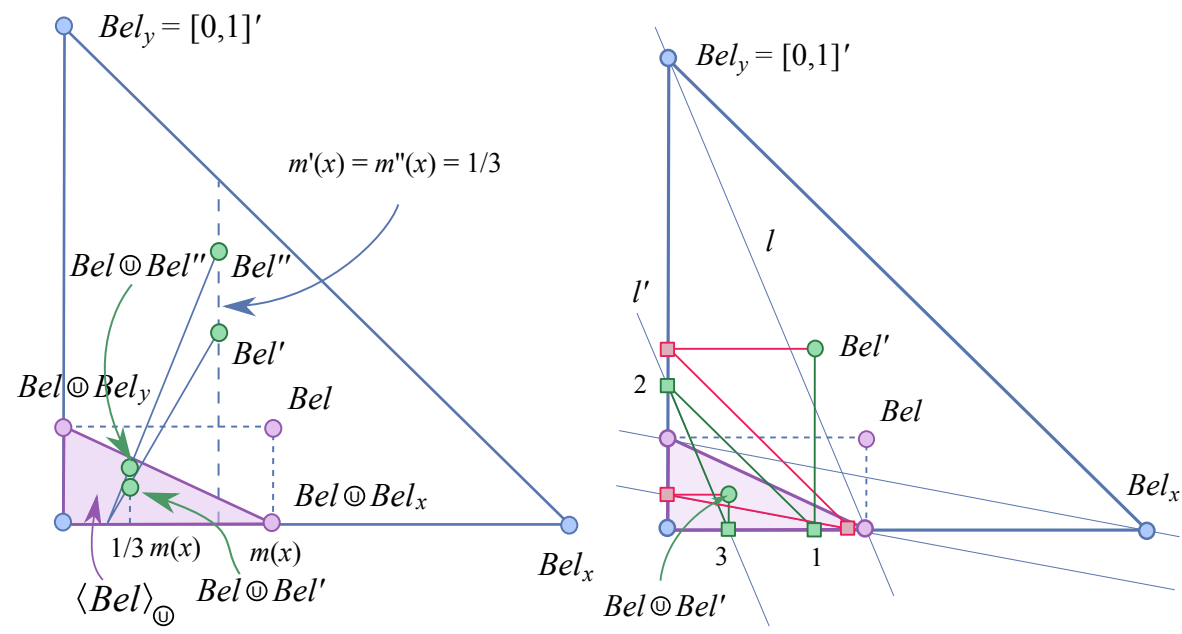

Fig. 3. (Left) Conditional subspace $\langle\mathrm{Bel}\rangle$ ()) for disjunctive combination on a binary frame. (Right) Geometric construction for the disjunctive combination of two belief functions $\mathrm{Bel}, \mathrm{Bel}^{\prime}$ on a binary frame.

Conditional subspace. By definition: $m_{(}(x)=m_{1}(x) m_{2}(x), m_{(}(y)=m_{1}(y) m_{2}(y)$, $m(\mathrm{C})(\Theta)=1-m_{1}(x) m_{2}(x)-m_{1}(y) m_{2}(y)$. Hence, in the usual vector notation:

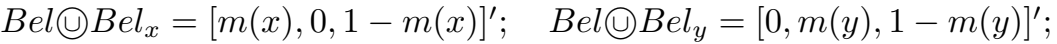

$$
\begin{aligned}
& \operatorname{Bel}\left(\text { ) } \operatorname{Bel}_{\Theta}=\operatorname{Bel}_{\Theta}\right. \text {. }
\end{aligned}
$$


The conditional subspace $\langle B e l\rangle($ () is thus the convex closure of the points (5):

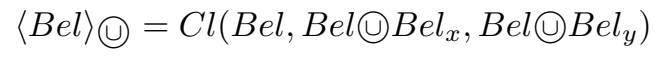

(see Figure 3). As in Yager's case: Bel@ $\left[\alpha B e l^{\prime}+(1-\alpha) B e l^{\prime \prime}\right]=\left[m(x)\left(\alpha m^{\prime}(x)+\right.\right.$ $\left.\left.(1-\alpha) m^{\prime \prime}(x)\right), m(y)\left(\alpha m^{\prime}(y)+(1-\alpha) m^{\prime \prime}(y)\right)\right]^{\prime}=\alpha B e l(0) B e l^{\prime}+(1-\alpha) B e l(0) B e l^{\prime \prime}$, i.e., (1) commutes with affine combination, at least in the binary case.

Pointwise behaviour. As in Yager's case, for disjunctive combination images of constant mass loci are parallel to each other. Actually, they are parallel to the corresponding constant mass loci and the coordinate axes (observe in Figure 3 (left) the locus $m^{\prime}(x)=m^{\prime \prime}(x)=1 / 3$ and its image in the conditional subspace $\langle B e l\rangle_{(\mathrm{C})}$, with coordinate $\left.1 / 3 m(x)\right)$. We can prove the following.

Theorem 1. In the binary case $\Theta=\{x, y\}$, all the lines joining Bel $l^{\prime}$ and Bel(ए)Bel' for any $\mathrm{Bel}^{\prime} \in \mathcal{B}$ intersect at the point:

$$
\overline{m(x)}=m^{\prime}(x) \frac{m(x)-m(y)}{1-m(y)}, \quad \overline{m(y)}=0 .
$$

Proof. Recalling the equation of the line joining two points $\left(\chi_{1}, v_{1}\right)$ and $\left(\chi_{2}, v_{2}\right)$ of $\mathbb{R}^{2}$, with coordinates $(\chi, v):\left(v-v_{1}\right)=\frac{v_{2}-v_{1}}{\chi_{2}-\chi_{1}}\left(\chi-\chi_{1}\right)$, we can identify the line joining $\mathrm{Bel}^{\prime}$ and $\mathrm{Bel(}\left(\mathrm{Bel} \mathrm{l}^{\prime}\right.$ as:

$$
\left(v-m^{\prime}(y)\right)=\frac{m(y) m^{\prime}(y)-m^{\prime}(y)}{m(x) m^{\prime}(x)-m^{\prime}(x)}\left(\chi-m^{\prime}(x)\right) .
$$

Its intersection with $v=0$ is the point (6), which does not depend on $m^{\prime}(y)$ (i.e., on the vertical location of $B e l^{\prime}$ on the constant mass loci).

A geometric construction for the disjunctive combination $\mathrm{Bel(}(\mathrm{Bel}$ ) of two $\mathrm{BFs}$ in $\mathcal{B}_{2}$ is provided by simple trigonometric arguments (Figure 3 (right)):

1. starting from $\mathrm{Bel}^{\prime}$, find its orthogonal projection onto the horizontal axis, with coordinate $m^{\prime}(x)$ (point 1);

2. draw the line with slope $45^{\circ}$ passing through such projection, and intersect it with the vertical axis, at coordinate $v=m^{\prime}(x)$ (point 2);

3. finally, take the line $l$ passing through $\operatorname{Bel}_{y}$ and the orthogonal projection of $B e l$ onto the horizontal axis, and draw a parallel one $l^{\prime}$ through point $2-$ its intersection with the horizontal axis (point 3 ) is the $x$ coordinate $m(x) m^{\prime}(x)$ of the desired combination.

A similar construction (in magenta) allows us to locate the $y$ coordinate of the combination (as shown in Figure 3 (right)).

\section{Combination of unnormalised belief functions}

In the case of unnormalised belief functions (those for which $m(\emptyset) \geq 0$, UBFs $[9])$, Dempster's rule is replaced by conjunctive combination: $m_{\circledast}(A) \doteq m_{\cap}(A)$. Disjunctive combination itself needs to be reassessed for UBFs as well. 
In the unnormalised case, a distinction exists between the belief measure $\operatorname{Bel}(A) \doteq \sum_{\emptyset \neq B \subset A} m(B)$ and the believability (in Smets' terminology) measure of an event $A$, denoted by: $b(A) \doteq \sum_{\emptyset \subset B \subset A} m(B)$. Here we analyse the geometric behavior of the latter, in which case $\emptyset$ is not treated as an exception: the case of belief measures is left to future work. As $b(\Theta)=1$, as usual, we neglect the related coordinate and represent believability functions as points of a Cartesian space of dimension $\left|2^{\Theta}\right|-1$ (as $\emptyset$ cannot be ignored anymore).

Conjunctive combination on the binary frame. In the case of a binary frame, the conjunctive combination of two belief functions $B e l_{1}$ and $B e l_{2}$ yields:

$$
\begin{aligned}
& m @(\emptyset)=m_{1}(\emptyset)+m_{2}(\emptyset)-m_{1}(\emptyset) m_{2}(\emptyset)+m_{1}(x) m_{2}(y)+m_{1}(y) m_{2}(x), \\
& m @(x)=m_{1}(x)\left(m_{2}(x)+m_{2}(\Theta)\right)+m_{1}(\Theta) m_{2}(x), \\
& m @(y)=m_{1}(y)\left(m_{2}(y)+m_{2}(\Theta)\right)+m_{1}(\Theta) m_{2}(y), \\
& m @(\Theta)=m_{1}(\Theta) m_{2}(\Theta) .
\end{aligned}
$$

Conditional subspace for conjunctive combination. The global behaviour of (a) in the binary (unnormalised) case can then be understood in terms of its conditional subspace, this time in $\mathbb{R}^{3}$. We have, after denoting $b=[b(\emptyset), b(x), b(y)]^{\prime}$ :

$$
\begin{aligned}
b @ b_{\emptyset} & =b_{\emptyset}=[1,1,1]^{\prime} ; \\
b @ b_{x} & =(m(\emptyset)+m(y)) b_{\emptyset}+(m(x)+m(\Theta)) b_{x} \\
& =[m(\emptyset)+m(y), 1, m(\emptyset)+m(y)]^{\prime}=b(y) b_{\emptyset}+(1-b(y)) b_{x} ; \\
b @ b_{y} & =(m(\emptyset)+m(x)) b_{\emptyset}+(m(y)+m(\Theta)) b_{y} \\
& =[m(\emptyset)+m(x), m(\emptyset)+m(x), 1]^{\prime}=b(x) b_{\emptyset}+(1-b(x)) b_{y} ; \\
b @ b_{\Theta} & =b,
\end{aligned}
$$

as $b_{x}=[0,1,0]^{\prime}, b_{y}=[0,0,1]^{\prime}, b_{\emptyset}=[1,1,1]^{\prime}$ and $b_{\Theta}=[0,0,0]^{\prime}$. From (8), we can note that the vertex $b @ b_{x}$ belongs to the line joining $b_{\emptyset}$ and $b_{x}$, with affine coordinate given by the believability assigned by $b$ to the other outcome $y$. Similarly, the vertex $b @ b_{y}$ belongs to the line joining $b_{\emptyset}$ and $b_{y}$, with coordinate given by the believability assigned by $b$ to outcome $x$ (see Figure 4 ).

Conditional subspace for disjunctive combination. As for the disjunctive combination, it is easy to see that in the unnormalised case we get: $b(0) b_{\Theta}=b_{\Theta}$, $b(0) b_{x}=b(x) b_{x}+(1-b(x)) b_{\Theta}, b(1) b_{\emptyset}=b, b(1) b_{y}=b(y) b_{y}+(1-b(y)) b_{\Theta}$, so that the conditional subspace is as in Figure 4. Note that, in the unnormalised case, there is a unit element to (C), namely $b_{\emptyset}$. We can observe a clear symmetry between the subspaces induced by disjunctive and conjunctive combination.

\section{Conclusions}

A number of questions remain open after this preliminary geometric analysis of other combination rules on binary spaces, and its extension to the case of unnormalised belief functions. In particular, the general pointwise geometric behaviour of disjunctive combination, in both the normalised and unnormalised case, needs to be understood. The question of whether disjunctive combination commutes with affine combination in general belief spaces remains open. A dual 
query concerns the conjuctive rule, as the alter ego of Dempster's rule in the unnormalised case. The general pointwise geometric behaviour of conjunctive and disjunctive combinations in the unnormalised case, as well as the complete description of their conditional subspaces, will also be subject of future work. The bold and cautious rules, which are also inherently defined for unnormalised belief functions, will also be analysed.

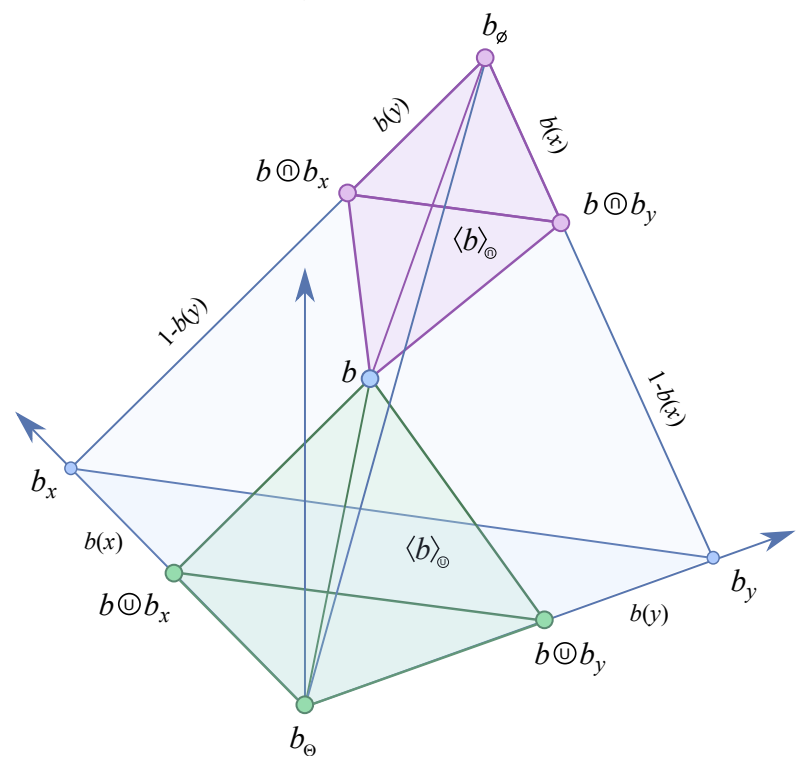

Fig. 4. Conditional subspaces induced by (-) and () in a binary frame, for the case of unnormalised belief functions.

\section{References}

1. Cuzzolin, F.: Geometry of Dempster's rule of combination. IEEE Transactions on Systems, Man and Cybernetics part B 34(2), 961-977 (2004)

2. Cuzzolin, F.: A geometric approach to the theory of evidence. IEEE Transactions on Systems, Man and Cybernetics part C 38(4), 522-534 (2008)

3. Cuzzolin, F.: The geometry of uncertainty. Springer-Verlag (2018)

4. Daniel, M.: Algebraic structures related to the combination of belief functions. Scientiae Mathematicae Japonicae 60(2), 501-511 (2004)

5. Dubois, D., Prade, H.: Representation and combination of uncertainty with belief functions and possibility measures. Comput Intell 4(3), 244-264 (1988)

6. Hajek, P., Valdes, J.J.: Generalized algebraic foundations of uncertainty processing in rule-based expert systems (dempsteroids). Comput Art Intel 10(1), 29-42 (1991)

7. Shafer, G.: A mathematical theory of evidence. Princeton University Press (1976)

8. Smets, P.: Belief functions : the disjunctive rule of combination and the generalized Bayesian theorem. International Journal of Approximate Reasoning 9, 1-35 (1993)

9. Smets, P.: The nature of the unnormalized beliefs encountered in the transferable belief model. In: Proceedings of UAI'92. pp. 292-297 (1992)

10. Yager, R.R.: On the Dempster-Shafer framework and new combination rules. Information Sciences 41(2), 93 - 137 (1987) 